\title{
Comportamiento de nematodos fitoparásitos en dos sistemas de cultivo de tomate convencional en Costa Rica*
}

\section{Plant parasitic nematodes behavior in two systems conventional tomato crop in Costa Rica}

Joaquín Durán-Mora¹, Tomás de Jesús Guzmán²

Fecha de recepción: 9 de agosto de 2016

Fecha de aprobación: 28 de setiembre de 2016

Durán-Mora, J; Guzmán, T. J. Comportamiento de nematodos fitoparásitos en dos sistemas de cultivo de tomate convencional en Costa Rica. Tecnología en Marcha. Vol. 30-3. Julio-Setiembre 2017. Pág 59-70.

DOI: 10.18845/tm.v30i3.3273

1 Ingeniero Agrónomo, Profesor e Investigador. Instituto Tecnológico de Costa Rica, Escuela de Agronomía, San Carlos, Costa Rica. Correo electrónico: jduran@itcr.ac.cr.

2 Ingeniero Agrónomo, Profesor e Investigador. Instituto Tecnológico de Costa Rica, Escuela de Agronomía, San Carlos, Costa Rica. Correo electrónico: tjguzman@itcr.ac.cr. Profesor tutor de tesis de doctorado. Doctorado en Ciencias Naturales para el Desarrollo (DOCINADE). 


\title{
Palabras claves
}

Tomate; café; monocultivo; nematodos; Meloidogyne; dinámica poblacional.

\section{Resumen}

Esta investigación se realizó en los cantones de Grecia (tomate asociado con café) y Heredia (tomate en monocultivo) de la Región Occidental de Costa Rica, durante setiembre del 2012 y febrero del 2013. Los muestreos mensuales consistieron en recolectar suelo y raíces de tomate en monocultivo y áreas agrícolas con café. Mediante el método centrifugación - flotación se extrajeron, identificaron y cuantificaron los nematodos presentes. Los géneros (en suelo y raíces) fueron Meloidogyne, Helicotylenchus, Criconemoides, Pratylenchus, Aphelenchus.

Los primeros meses después del trasplante (DDT) las poblaciones de fitonematodos fueron bajas. A partir del cuarto mes DDT en áreas en asocio, las muestras de suelo sin oxamil, presentaron aumentos en poblaciones de Meloidogyne (48 a 7300 individuos) y Helicotylenchus (234 a 2100), mientras que en áreas con oxamil, las poblaciones de Meloidogyne se incrementaron de 50 a 2800. En raíces de tomate de áreas sin oxamil, se incrementaron las poblacionales de Meloidogyne (1625 individuos a 59200) y Helicotylenchus (2175 a 3800); y en zonas con oxamil, las poblacionales de Meloidogyne aumentaron de 3288 a 68467.

El tomate en monocultivo, a partir del cuarto mes DDT no presentó poblaciones de Meloidogyne en suelo, mientras que Helicotylenchus varía de 97 a 123. En raíces, las poblaciones de Meloidogyne varían de 17 a 52, y para Helicotylenchus de 405 a 392.

La presencia de agallas por Meloidogyne se hace más evidente al asociarse tomate con café, aumentando los niveles hasta el 90\%. En áreas de tomate en monocultivo no se presentó agallas ni poblaciones de Meloidogyne.

\section{Keywords}

Tomatoes; coffee; monoculture; nematodes; Meloidogyne; population dynamics.

\begin{abstract}
This research was conducted in the cantons of Grecia (tomato in associated with coffee) and Heredia (tomato monoculture) in the Western Region of Costa Rica, during September 2012 and February 2013. The monthly sampling consisted of collecting soil and tomato roots in monoculture and agricultural areas with coffee, and by centrifugation method - flotation extracted, identified and quantified the nematodes. The nematodes (soil and roots) were Meloidogyne, Helicotylenchus, Criconemoides, Pratylenchus, Aphelenchus.

The first few months after transplantation (DDT) populations of plant parasitic nematodes were low. From the fourth month DDT in areas in partnership, soil samples without oxamyl showed increases in populations of Meloidogyne (48-7.300 individuals) and Helicotylenchus (234-2.100), whereas in areas with oxamyl, Meloidogyne increase of 50 individuals roots 2.800. In tomato roots without oxamil areas, the population of Meloidogyne (1.625 individuals to 59.200) and Helicotylenchus (2.175-3.800) increased; and in areas with oxamyl, population of Meloidogyne increased from 3.288 to 68.467 .

Tomato monoculture, from the fourth month DDT did not present populations of Meloidogyne in soil, while Helicotylenchus varies from 97 to 123. In roots, Meloidogyne populations vary from 17 to 52, and Helicotylenchus of 405-392.
\end{abstract}


The presence of Meloidogyne galls becomes more evident by partnering with tomato coffee, increasing levels up to $90 \%$. In areas tomato monoculture not have the galls or populations of Meloidogyne was presented.

\section{Introducción}

Para solventar la pobreza y la producción de alimentos, el cambio agrícola ha recurrido al incremento de densidades de siembra, monocultivos y uso intensivo de productos fitosanitarios, ocasionando un desequilibrio ecológico, el resurgimiento de plagas y enfermedades, generando dificultad en su combate y pérdidas en cosechas.

En Costa Rica, los productos hortícolas forman parte de la canasta básica, siendo el tomate (Solanum lycopersicum L., sin. Lycopersicon esculentum Mill.) uno de los cultivos de mayor importancia económica y social para un cierto grupo de agricultores, que demanda para su producción diversas labores de manejo y mantenimiento, solventándose con mano de obra familiar y en muchos casos poco calificada.

Al 2014, existían en Costa Rica 1.250,9 hectáreas dedicadas a la siembra de tomate, de las cuales cerca de 562,5 corresponden a la zona Central Occidental (provincias de Alajuela y Heredia), constituyéndose en uno de los cultivos de exportación de mayor importancia, aumentando las exportaciones de fruta madura (fresca o refrigerada, concentrados, purés, salsas) de 14.108,0 t en 2005 a 54.093,4 t en 2008 [1], y disminuyendo $13.154 \mathrm{~kg}$ durante el 2012 lo que generó ingresos alrededor \$24.500 en tomates frescos [2].

Los fitonematodos son organismos que afectan en distinto grado una amplia variedad de cultivos, en especial los hortícolas, causando graves daños económicos al disminuir la productividad. A nivel mundial existen una gran cantidad de nematodos, que causan pérdidas hasta del 38\% [3], mientras que autores citados por Hassan et al. [4] estiman reducciones entre el 28 y 68\%. De la diversidad de géneros fitopatógenos están Meloidogyne sp., Pratylenchus sp., Ditylenchus sp., Heterodera sp., Tylenchus sp. [5]. Al ser de tamaño reducido (0,2 mm), y completar su ciclo de vida bajo el suelo, sus principales daños a nivel radical son de difícil diagnóstico en las primeras etapas de infección. En etapas avanzadas, producen obstrucción de vasos e impiden la absorción de sustancias por las raíces, observándose necrosis radical, clorosis, enanismo, amarillamiento de partes aéreas, menor desarrollo de la planta y pérdida significativa de frutos, y en casos extremos, la muerte de la planta. Por lo general, interaccionan con otros patógenos, mediante las heridas facilitando la entrada de bacterias, hongos y virus.

En tomate se reconocen los géneros Meloidogyne, Pratylenchus, Rotylenchulus y Nacobbus como los más dañinos [6], de los cuales el género que ocasiona mayores pérdidas en la producción lo constituye Meloidogyne, conocido como nematodo agallador de las raíces, ya que su forma de expresión es la producción de abultamientos típicos radicales en forma de agallas. Meloidogyne es uno de los géneros de mayor importancia económica y comprende más de 80 especies diferentes con un amplio rango de plantas huésped (más de 5000 especies de plantas) y una distribución mundial [7].

Algunas especies de Meloidogyne detectadas en Costa Rica son incognita, javanica, hapla, salasi, fallax y chiwoodi, suponiéndose que éstas últimas ingresaron al país con material vegetativo contaminado, pero por sus características morfológicas muy similares, las especies de Meloidogyne presentan problemas en el momento de la identificación. A pesar de la presencia de estos nematodos en varios hospederos y del daño potencial que ocasionan, la información cuantificable de los daños que provocan prácticamente es inexistente [8]. 
Según Fernández y Quesada [9], el cultivo del tomate se encuentra en asocio con las especies de Criconemella sp., Helicotylenchus sp., Hemicycliophora sp., Meloidogyne hapla, Meloidogyne incognita, Meloidogyne javanica, Pratylenchus sp., Trichodorus sp.

En la Zona Occidental de Costa Rica, muchas de las plantaciones temporales de tomate, son establecidas en monocultivo o en asocio a café (Coffea arabica), aprovechando las condiciones de suelo, manejo y disponibilidad de zonas agrícolas. La necesidad de ampliar horizontes con el fin de que el agricultor reciba mejores beneficios económicos, ha transformado este tipo de cultivos en explotaciones altamente tecnificadas, demandantes de insumos externos y productos sintéticos, de altos costos y peligros ambientales, principalmente en zonas rurales.

La identificación precisa de los nematodos es esencial para desarrollar cualquier estudio fitosanitario y comprender el efecto que causan en los cultivos y el entorno donde se desarrollan. Además, la determinación de los niveles críticos y los umbrales de acción permite contar con varios parámetros para un manejo adecuado, establecer estrategias de manejo y control adecuadas, buscando un manejo más sostenible.

Por tanto, esta investigación se realizó con el objetivo de analizar el comportamiento de distintas especies de nematodos presentes en el cultivo del tomate convencional en dos sistemas de cultivo en la Zona Central Occidental de Costa Rica.

\section{Materiales y Métodos}

El área de investigación se desarrolló en los cantones de Grecia (Rincón de Arias) y Heredia (Santa Rosa) de la Región Occidental de Costa Rica (cuadro 1), entre los meses de setiembre 2012 y febrero de 2013. Como práctica agrícola, los agricultores, establecen el cultivo de tomate entre las hileras del cafetal recién podado con el fin de aprovechar el espacio dentro de las plantaciones, brindando el mantenimiento necesario a los cafetales y al cultivo del tomate.

Las áreas muestreadas fueron plantaciones de tomate en monocultivo y en asocio con café, en las cuales utilizaban riego por goteo mediante el cual se le suministraba semanalmente el paquete fitosanitario de fertilizantes y plaguicidas al suelo. Para el control de nematodos se usa normalmente el nematicida oxamil (24 SL, 2-5 litros/ha), el cual se recomienda su aplicación entre los 25 y 30 días después del trasplante. A nivel de follaje, el manejo sanitario se realizó en forma preventiva mediante aplicaciones con aspersores y mangueras provenientes del pulverizador hidráulico acoplado al tractor.

La plantación de tomate en monocultivo fueron áreas de barbecho, mientras que la plantación de café, presentaba un ciclo de poda de renovación. Las áreas muestreadas se referenciaron mediante sistemas de posicionamiento geográfico (GPS).

\section{Muestreo de campo}

Se recolectaron muestras de suelo y raíces, desde uno y hasta seis meses después del trasplante, a una profundidad de 20 centímetros. Las muestras recolectadas, se acondicionaron en bolsas plásticas con la debida rotulación, y transportadas para el posterior análisis al Laboratorio de Nematología del Instituto Tecnológico de Costa Rica, Sede Santa Clara (Costa Rica).

\section{Análisis de muestras en Laboratorio}

Cada muestra de campo se mezcló y homogenizó, para la cuantificación, determinación e identificación de las poblaciones juveniles y adultos de los fitonematodos. Las muestras de suelo y raíces se procesaron por el método de centrifugación-flotación [10]. 


\section{Sintomatología radical}

Se pesaron y lavaron 25 gramos de raíces con agua limpia sobre una malla para evitar la pérdida radical, posteriormente se determinó la escala radical (cuadro 2).

Cuadro 1. Características generales y grado de asocio de las fincas tomateras consideradas en la investigación. Costa Rica, 2012-2013.

\begin{tabular}{|c|c|c|}
\hline Características de las fincas & Grecia & Heredia \\
\hline Ubicación & Rincón de Arias & Santa Rosa \\
\hline Altura sobre el nivel del mar (msnm) & 950 & 1118 \\
\hline Precipitación anual (mm) & 2491 & 2500 \\
\hline Temperatura promedio $\left({ }^{\circ} \mathrm{C}\right)$ & 20 & 25,6 \\
\hline Coordenadas & $10^{\circ} 4^{\prime} \mathrm{N}, 84^{\circ} 18^{\prime} \mathrm{O}$ & $09^{\circ} 58^{\prime} \mathrm{N}, 84^{\circ} 05^{\prime} \mathrm{O}$ \\
\hline Trasplante cultivo & 7 agosto 2012 & 17 agosto 2012 \\
\hline Aplicación de oxamil (24 SL) & 11 setiembre 2012 & 1 setiembre 2012 \\
\hline Asociación & $\begin{array}{c}\text { Tomate y Café } \\
\text { Parcela } 1 \text { (sin oxamil), } \\
\text { Parcela } 2 \text { (con oxamil) }\end{array}$ & $\begin{array}{c}\text { Tomate en monocultivo } \\
\text { (Parcela 3) }\end{array}$ \\
\hline
\end{tabular}

Cuadro 2. Sintomatología radical del cultivo utilizando la evaluación del índice de severidad de agallas (Adaptado de Baker [11]).

\begin{tabular}{|c|c|c|}
\hline Escala & Criterio de Agallamiento & Sintomatología (\%) \\
\hline 0 & Raíz sana & Raíz sana \\
\hline 1 & Muy leve & 1 al 10 \\
\hline 2 & Leve & 11 al 20 \\
\hline 3 & Media & 21 al 40 \\
\hline 4 & Moderada & 41 al 70 \\
\hline 5 & Alta & 71 al 90 \\
\hline 6 & Extrema & 91 al 100 \\
\hline
\end{tabular}

Análisis de muestras de raíces

Las raíces se lavaron y cortaron en trozos pequeños. Se pesaron 25 gramos y licuaron en dos tiempos de 10 segundos, a velocidad lenta y rápida. La separación final de los nematodos se realizó decantando la solución obtenida sobre un juego de cribas superpuesto de 100 y 400 mallas. El material contenido en la criba de 400 mallas fue centrifugado por 5 minutos a 3000 rpm, después de descartar el sobrenadante se resuspendió el precipitado en solución azucarada (1,18 de gravedad específica) y se centrifugó nuevamente por 5 minutos a 3000 rpm.

El sobrenadante conteniendo los nematodos se decantó sobre una criba de 400 mallas, lavándose el exceso de azúcar con suficiente agua. Finalmente se procedió a colectar los nematodos del tamiz lavando con la ayuda de una piceta, aforando la muestra a $10 \mathrm{ml}$ de los cuales se colocaron $3 \mathrm{ml}$ en una placa cuenta nematodos (tipo Neubauer). 
Análisis de muestras de suelo

Las muestras fueron homogenizadas y submuestras de 100 gramos fueron colocadas en un balde y se les realizaron dos lavados seguidos de un minuto de suspensión cada uno.

La separación final de los nematodos se realizó decantando la solución obtenida sobre un juego de cribas superpuesto de 100 y 400 mallas. El material contenido en la criba de 400 mallas fue centrifugado por 5 minutos a 3000 rpm, después de descartar el sobrenadante se resuspendió el precipitado en solución azucarada (1,18 de gravedad específica) y se centrifugó nuevamente por 5 minutos a 3000 rpm.

El sobrenadante conteniendo los nematodos se decantó sobre una criba de 400 mallas, lavándose el exceso de azúcar con suficiente agua. Finalmente se procedió a colectar los nematodos del tamiz lavando con la ayuda de una piceta, aforando la muestra a $10 \mathrm{ml}$ de los cuales se colocaron $3 \mathrm{ml}$ en una placa cuenta nematodos (tipo Neubauer).

Identificación de nematodos

En ambos procesos de extracción, los nematodos fueron cuantificados con la ayuda de un microscopio invertido (40x), e identificados con la ayuda de claves dicotómicas de Mai et al. [12], el Manual de Fitopatología de Marbán [13], Manual de identificación de géneros de nematodos importantes en Costa Rica de Esquivel [10], Manual de Laboratorio de Nematodos de Plantas de Zuckerman et al. [14], y el Índice llustrado de los descriptores de nematodos parásitos de plantas del Commonwealth Institute of Helminthology [15].

\section{Dinámicas Poblacionales}

Se muestreó mensualmente las fincas de tomate seleccionadas. En cada una de las áreas se recolectó suelo y material radical de plantas que muestren alguna sintomatología (poco desarrollo, clorosis, agallas radicales, etc.) así como de plantas aparentemente sanas. A nivel radical se analizó esta sintomatología utilizando inicialmente las raíces sanas y funcionales, así como daños a nivel radical los cuales fueron cuantificados mediante la elaboración de escalas de severidad asociadas a cada sintomatología. Con los valores poblacionales se elaboraron las gráficas de dinámicas respectivas.

\section{Resultados}

En esta investigación, tanto en suelo como en raíces de tomate se identificaron las especies de fitonematodos Meloidogyne, Helicotylenchus, Criconemoides, Pratylenchus y Aphelenchus. Se consideran de mayor importancia Meloidogyne y Helicotylenchus, por sus niveles poblacionales a lo largo del ciclo del cultivo.

\section{Sintomatología radical}

Los daños a nivel radical en el cultivo del tomate fueron evaluados usando el índice de severidad de agallas. En el cuadro 3, se aprecia que desde el tercer mes después del trasplante se empiezan a dar evidencia de la presencia de agallas radicales. Las parcelas en las cuales estaba asociado el tomate con café presentaron los mayores niveles de agallas (hasta del $90 \%$ ), mientras que la parcela del tomate en monocultivo no presentó agallas ni poblaciones de Meloidogyne durante el periodo de evaluación. Esta ausencia de agallas se puede asociar al periodo en barbecho (cerca de 3 años) existente antes de la siembra del monocultivo, siendo predominantes las malezas gramíneas.

Este aumento del agallamiento, se puede atribuir a la pérdida de la efectividad del nematicida químico en el tiempo, ya que estos valores se incrementan cerca de 4 meses después del trasplante (3 meses después de la aplicación en el sistema de riego por goteo). 
Cuadro 3. Porcentaje de agallas radicales en plantaciones de tomate en monocultivo y en asocio con café. Costa Rica, 2012-2013.

\begin{tabular}{|c|c|c|c|c|c|c|c|c|}
\hline \multirow{3}{*}{ Criterio / Parcela } & \multicolumn{8}{|c|}{$\begin{array}{l}\text { Porcentaje de agallamiento } \\
\text { (peso raíces agalladas/peso total raíces) }\end{array}$} \\
\hline & \multicolumn{2}{|c|}{ mdt * } & \multicolumn{2}{|r|}{$4 \mathrm{mdt}$} & \multicolumn{2}{|r|}{$5 \mathrm{mdt}$} & \multicolumn{2}{|r|}{$6 \mathrm{mdt}$} \\
\hline & $\%$ & Escala & $\%$ & Escala & $\%$ & Escala & $\%$ & Escala \\
\hline $\begin{array}{c}\text { Parcela } 1 \text { (sin oxamil, tomate asociado } \\
\text { con café) }\end{array}$ & 0,05 & 0 & 30 & 3 & 90 & 5 & 90 & 5 \\
\hline $\begin{array}{c}\text { Parcela } 2 \text { (con oxamil, tomate asociado } \\
\text { con café) }\end{array}$ & 0,05 & 0 & 90 & 5 & 17 & 2 & 90 & 5 \\
\hline Parcela 3 (tomate en monocultivo) & 0,05 & 0 & 0 & 0 & 0 & 0 & 0 & 0 \\
\hline
\end{tabular}

*: mdt (meses después del trasplante). Evaluación 1 y 2 mdt no presentaron agallamiento.

\section{Dinámicas poblacionales}

A continuación, se presentan los resultados de la identificación y cuantificación de los principales nematodos observados en el cultivo del tomate.

Primer muestreo, 1 mes después del trasplante

En esta fecha, sólo la finca establecida con tomate en asocio con café (Parcela 1 y 2) en Grecia, estaba en edad fenológica para realizar las extracciones de nematodos.

En las muestras extraídas de suelo, se aprecia que las áreas aplicadas con nematicida presentaron menores poblacionales para los nematodos Meloidogyne, Helicotylenchus, Criconemoides, Pratylenchus y Aphelenchus comparadas con las áreas sin aplicación (figura 1).
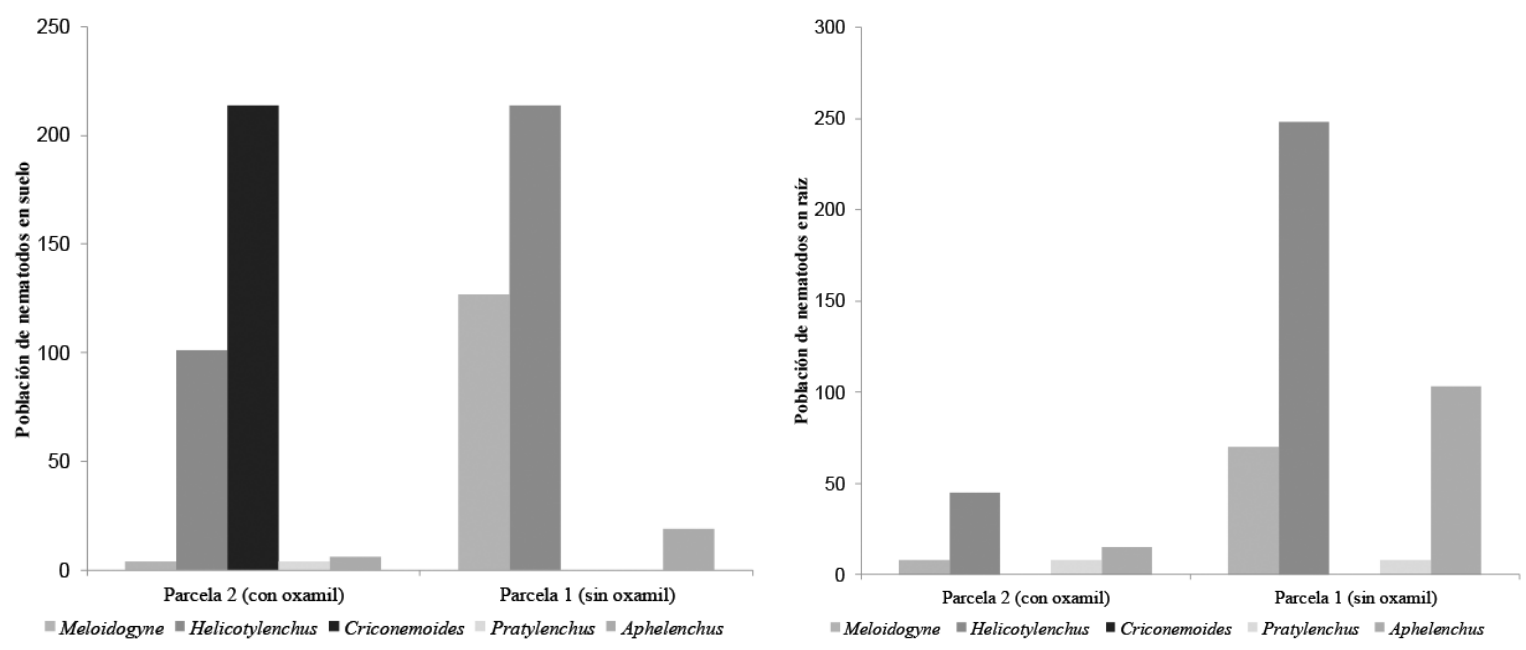

Figura 1. Poblaciones de nematodos presentes en suelo y raíces de plantaciones de tomate.Un mes después del trasplante. Setiembre 2012.

En raíces, se presenta un comportamiento similar a los nematodos de suelo, excepto con Criconemoides, en la cual no se encontraron nematodos en las áreas muestreadas (figura 1). 
Segundo muestreo, 2 meses después del trasplante

Ambas fincas presentaron poblaciones importantes de fitonematodos. Helicotylenchus, al ser ectoparásito le da mayor sobrevivencia por su movilidad ya que incluso en las áreas con aplicaciones de nematicidas y la poca residualidad del plaguicida en el suelo, hace que las poblaciones puedan incrementarse. El efecto del nematicida sistémico provoca que, a nivel interno de las raíces, las cantidades de nematodos tiendan a reducirse en niveles importantes, creando un efecto de control al menos por un periodo de un mes (figura 2).
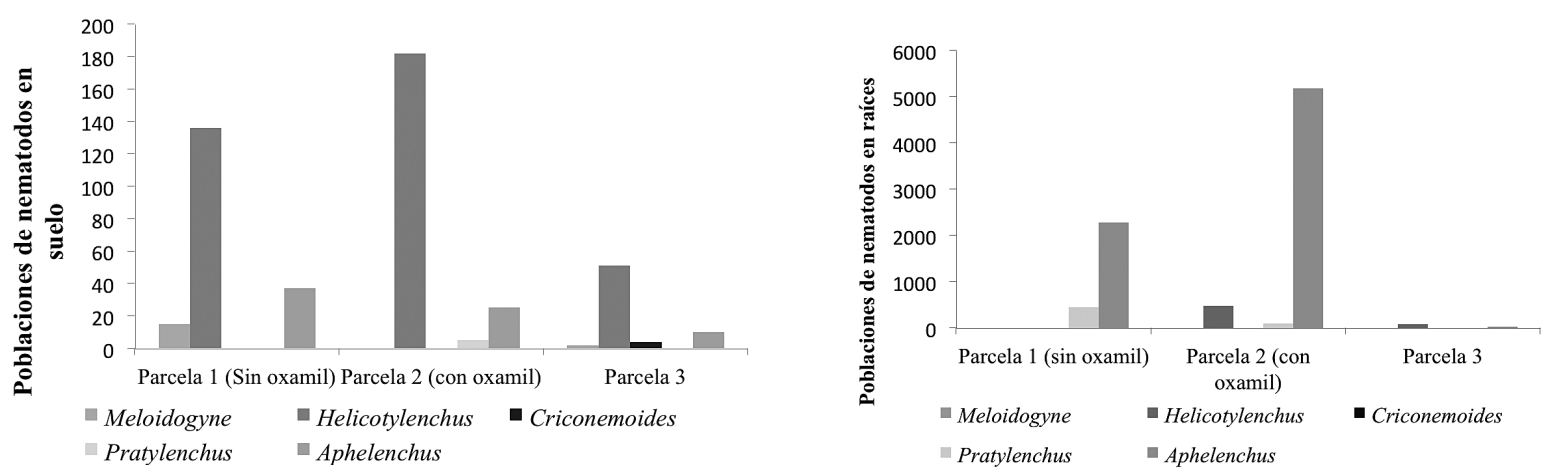

Figura 2. Poblaciones de nematodos presentes en suelo y raíces de plantaciones de tomate. Dos meses después del trasplante. Octubre 2012.

En las muestras de raíces (figura 2), las poblaciones de Aphelenchus y Helicotylenchus se presentan por encima de los otros géneros de nematodos, a pesar de que las cantidades de raíces funcionales no sean muy altos.

Tercer muestreo, 3 meses después del trasplante

En esta etapa, se había iniciado la cosecha de los frutos y es normal que las plantas necesiten más requerimentos de nutrición y agua, principalmente. El aumento en la cantidad de raíces, crea el espacio necesario para que se incrementen considerablente las poblaciones de nematodos. En la figura 3 se aprecia que las poblacionales de Helicotylenchus a nivel de suelo han aumentado sustancialmente respecto al mes anterior, sin embargo no ha sido similar con los otros nematodos.

A nivel de suelo, la parcela con oxamil (Parcela 1), presenta 597 individuos de Helicotylenchus y 90 de Meloidogyne. Mientras que la parcela 2 al encontrarse sin cobertura boscosa cercana, y al estar completamente expuesta a la radiación solar, se crea un ambiente en la cual puede darse un aumento leve de poblaciones de nematodos.

En el muestreo de raíces, las poblaciones de Helicotylenchus están bastante elevadas (7.488) en las áreas de que se utilizó nematicida químico comparadas con los otros nematodos, en especial Meloidogyne, nematodo de mayor importancia en el cultivo del tomate (figura 3).

Cuarto muestreo, 4 meses después del trasplante

Los niveles de fitonematodos en suelo han aumentado respecto al muestreo anterior, posiblemente por la pérdida del efecto nematicida, aunado al uso de las enmiendas aplicadas con el riego por goteo. Las poblaciones de Helicotylenchus en suelo se encuentran en mayor cantidad en las parcelas sin aplicación de nematicida (234 individuos), comparadas con las parcelas con nematicida (63) y tomate sin asocio (51) (figura 4). 


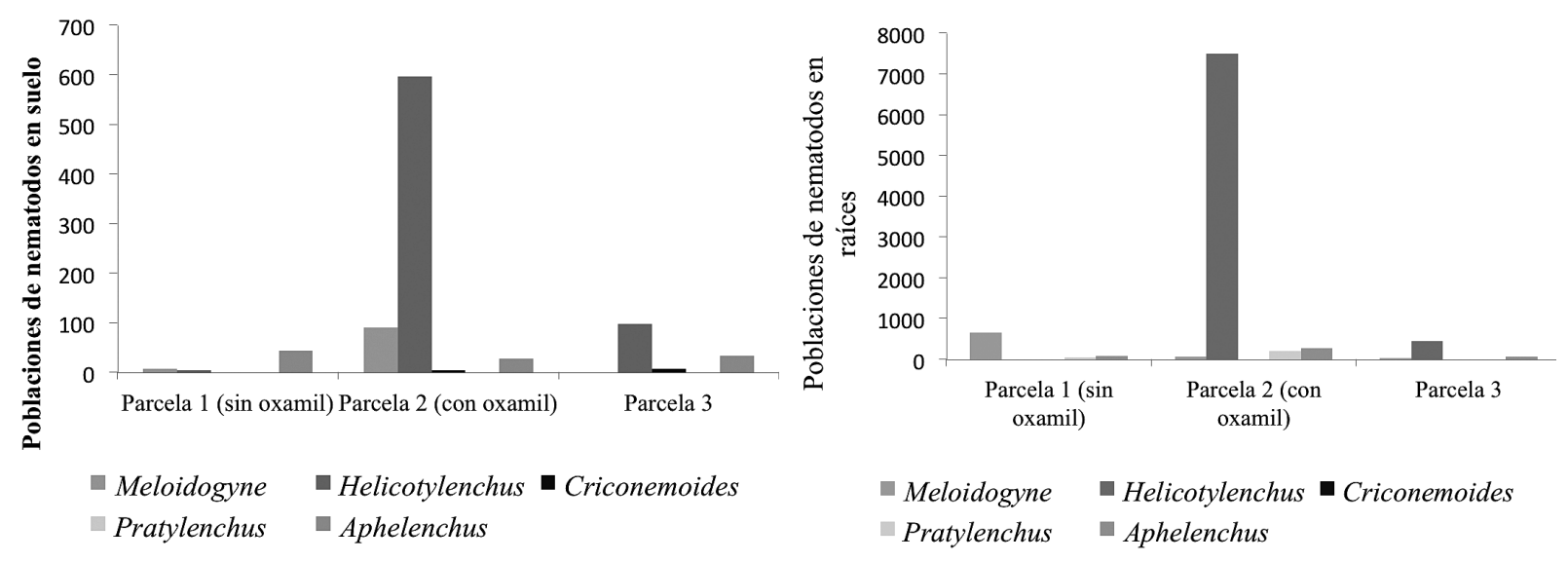

Figura 3. Poblaciones de nematodos presentes en suelo y raíces de plantaciones de tomate. Tres meses después del trasplante. Noviembre 2012.
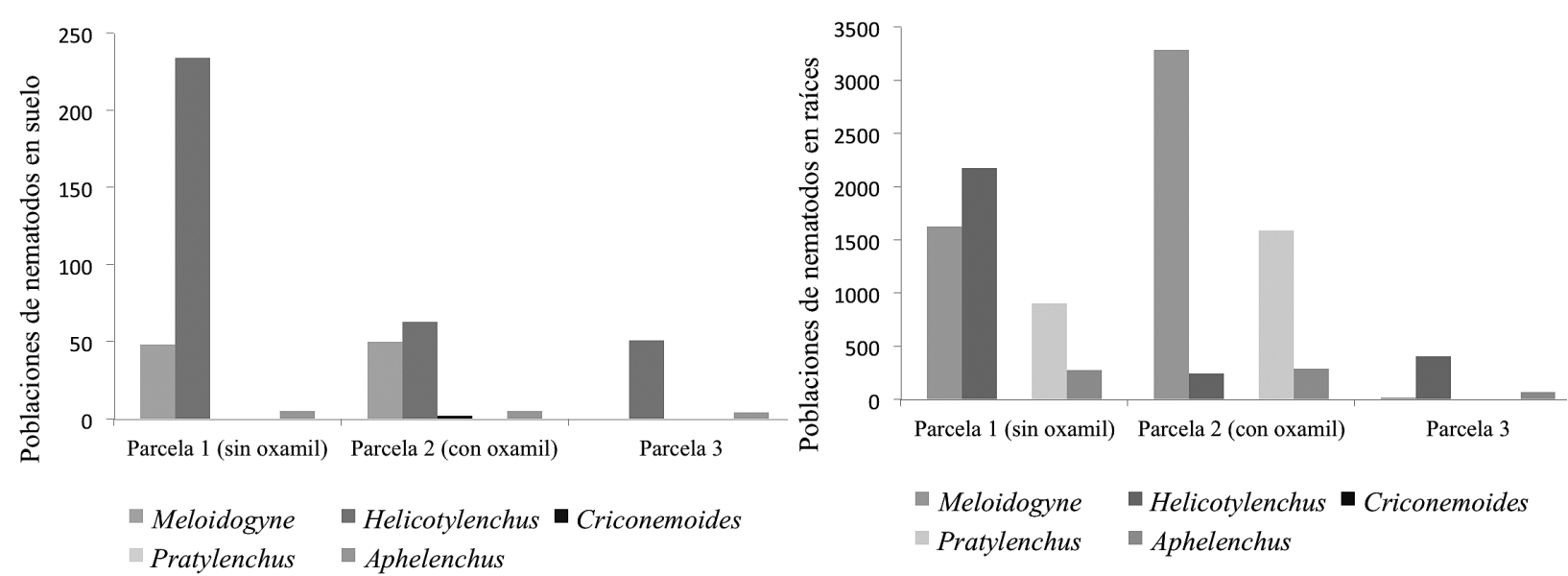

Figura 4. Poblaciones de nematodos presentes en suelo y raíces de plantaciones de tomate.Cuatro meses después del trasplante. Diciembre 2012.

En este muestreo se cuantificó una cantidad de nematodos en raíces mayor que el mes anterior (Parcela 2, con oxamil), pero disminuyó en la Parcela 1 (sin oxamil) y Parcela 3 (cultivo solo) posiblemente al reducirse el efecto del nematicida. Los niveles de nematodos en raíces en Parcela 3 (tomate en monocultivo), no presentan valores considerables de nematodos.

Quinto muestreo, 5 meses después del trasplante

Los niveles poblacionales de Meloidogyne se incrementaron debido a ser unas de las últimas etapas de cosecha y la consecuente disminución del manejo de plagas. A nivel de suelo (figura 5), en las parcelas sin nematicida se determinaron 3.100 individuos, mientras que las áreas con nematicidas se cuantificaron 220.

En raíces se determinaron 10444 individuos de Meloidogyne en parcelas sin nematicida y de 993 en parcelas con nematicida. Las poblaciones de nematodos en la parcela de tomate sin asocio son muy bajas (figura 5). 

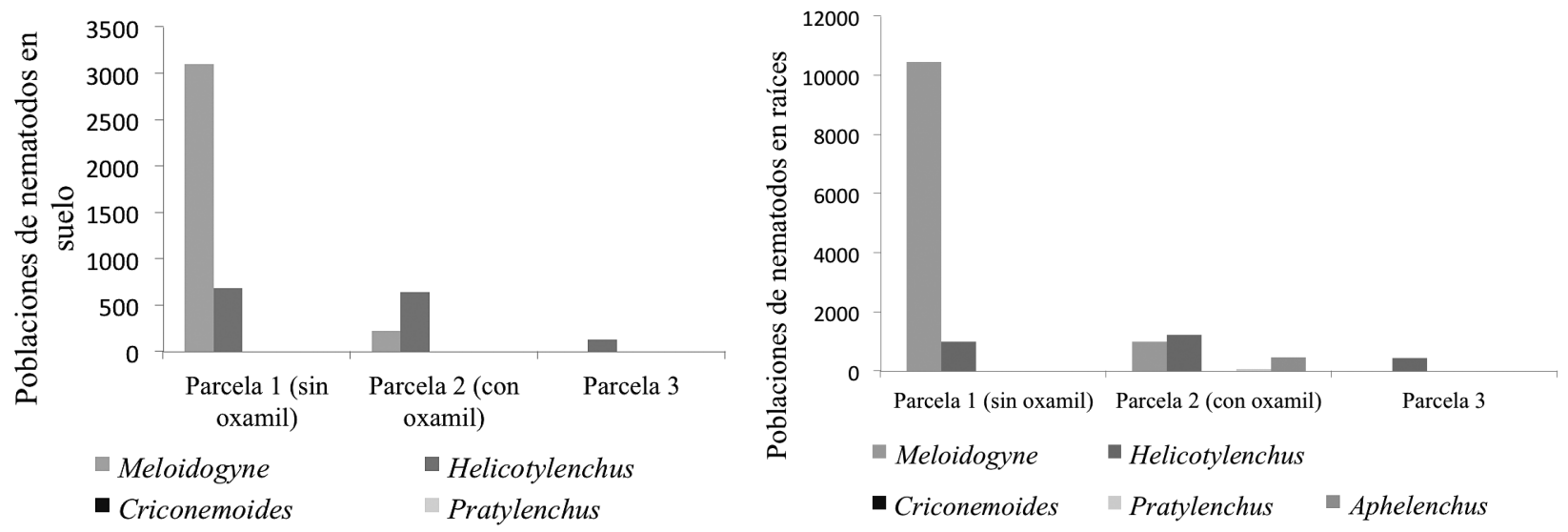

Figura 5. Poblaciones de nematodos presentes en suelo y raíces de plantaciones de tomate. Cinco meses después del trasplante. Enero 2013.

\section{Sexto muestreo, 6 meses después del trasplante}

Para este muestreo, las prácticas agronómicas en el cultivo se han reducido debido a que el pico de producción ya fue superado, y no justifica realizar manejo de plagas y fertilización.

A partir de esta fecha, los niveles poblacionales de Meloidogyne tanto en suelo como en raíces de la Parcela 1 se incrementaron considerablemente. En suelo se determinaron 7300 y 2800 individuos en las parcelas sin (Parcela 1) y con (Parcela 2) oxamil, respectivamente. En raíces se cuantificaron 59200 individuos en las parcelas sin nematicidas (Parcela 1), y 68467 individuos en las parcelas con nematicidas (Parcela 21) (figura 6).
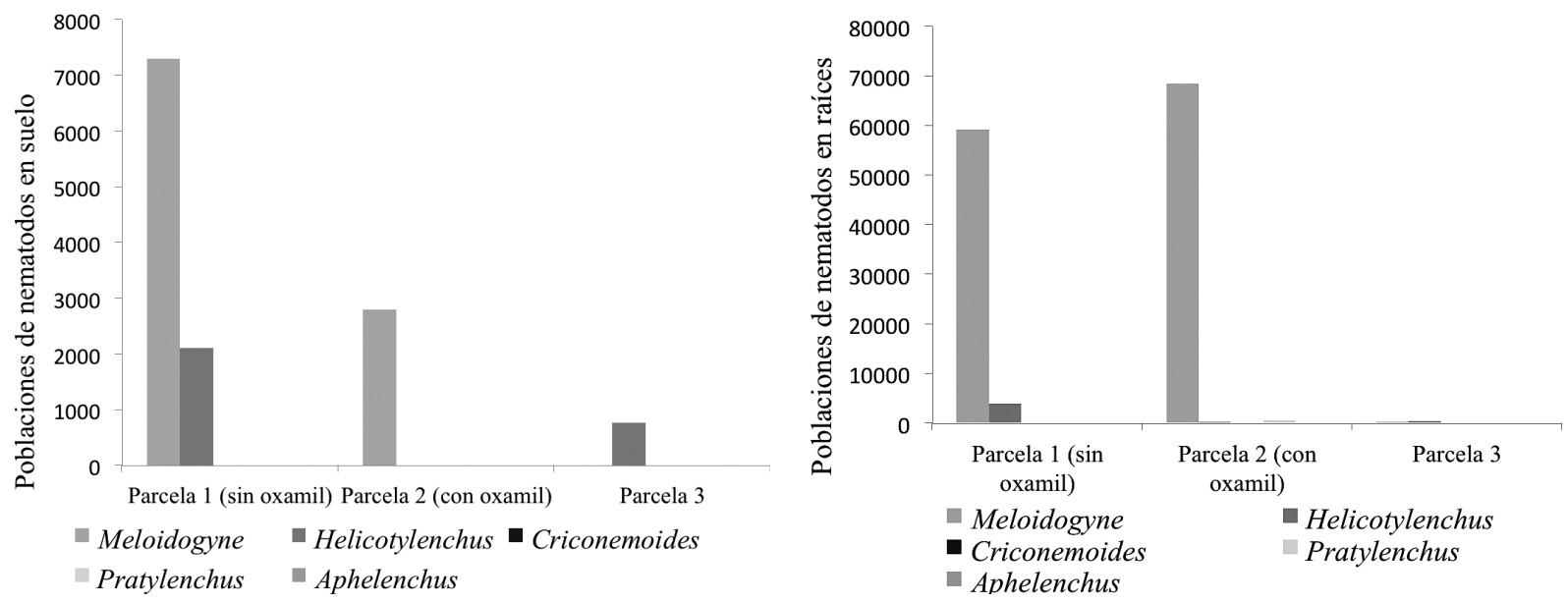

Figura 6. Poblaciones de nematodos presentes en suelo y raíces de plantaciones de tomate. Seis meses después del trasplante. Febrero 2013.

En la parcela de tomate en monocultivo, se cuantificaron poblaciones de 52 individuos de Meloidogyne y 392 Helicotylenchus.

La finca en la cual el cultivo del tomate se estableció sin asocio, presentó cantidades de nematodos relativamente bajos comparados con las parcelas en asocio con café. 


\section{Conclusiones}

Con base en los resultados obtenidos en esta investigación se puede analizar que:

1. La aplicación de nematicidas mantiene bajos los niveles de nematodos por espacio de 2 meses, especialmente Meloidogyne.

2. Los niveles de agallas en las parcelas con tomate en asocio fueron del $90 \%$, por el efecto de las poblaciones de Meloidogyne. La parcela del tomate en monocultivo no presentó agallas, por un posible efecto del barbecho al romper el ciclo biológico de los nematodos.

3. Las especies de fitonematodos identificadas tanto en suelo como en raíces de tomate fueron Meloidogyne, Helicotylenchus, Criconemoides, Pratylenchus y Aphelenchus.

4. En los muestreos de suelo sin aplicación de nematicidas, a partir del cuarto mes después del trasplante las poblaciones de Meloidogyne y Helicotylenchus aumentan considerablemente de 48 individuos a 7300, y de 234 a 2100, respectivamente.

5. En las muestras de suelo con aplicación de nematicidas, a partir del cuarto mes después del trasplante las poblaciones de Meloidogyne aumentan de 50 individuos a 2800, mientras que Helicotylenchus disminuye de 263 a 0.

6. En las muestras de raíces de tomate sin nematicidas, a partir del cuarto mes después del trasplante las poblaciones de Meloidogyne y Helicotylenchus aumentan considerablemente de 1625 individuos a 59200, y de 2175 a 3800, respectivamente.

7. En raíces con aplicación de nematicidas, a partir del cuarto mes después del trasplante estos valores poblacionales de Meloidogyne de 3288 individuos a 68467; en el caso de Helicotylenchus disminuye de 240 a 67.

8. Las áreas de suelo en el cultivo del tomate en monocultivo, a partir del cuarto mes después del trasplante no presentan poblaciones de Meloidogyne y para Helicotylenchus varía de 97 individuos a 123.

9. En las áreas de raíz en el cultivo del tomate en monocultivo, a partir del cuarto mes después del trasplante las poblaciones de Meloidogyne varían de 17 a 52 individuos, y para Helicotylenchus de 405 a 392.

\section{Recomendaciones}

Por la existencia de pocos estudios sobre el comportamiento poblacional, conviene extender estos estudios por al menos tres ciclos de cultivo en diversas zonas cultivables.

Establecer parcelas de monitoreo en campo a través del tiempo para establecer los niveles poblacionales en áreas de monocultivo de tomate, y en áreas cultivadas de café en asocio con tomate.

\section{Referencias}

[1] Ministerio de Agricultura y Ganadería, «Agrocadena de Tomate,» de Agrocadena de Tomate, San José, MAG, 2009, p. 80.

[2] Ministerio de Agricultura y Ganadería, « http://www.infoagro.go.cr,» 6 junio 2016. [En línea]. Available: http:// www.infoagro.go.cr/AgroNoticias/Paginas/Exportación de chile y tomate.aspx. .

[3] R. Sikora y E. Fernández, «Nematode Parasites of Vegetables, de Plant Parasitic Nematodes in Subtropical and Tropical Agriculture, UK, CABI Publishing, 2005, pp. 319-392. 
[4] M. Hassan, P. Chindo, P. Marley y M. Alegbej, «Management of root knot nematodes (Meloidogyne spp.) on tomato (Lycopersicon lycopersicum) using organic wastes in Zaria, Nigeria,» Plant Protect Sci, vol. 46, $n^{\circ} 1$, pp. 34-38, 2010.

[5] Meister Media Worldwide, Plagas y Enfermedades: Guía de Identificación y Manejo del Tomate, North Caroline: North Caroline State University; 2009.

[6] Centro Agronómico Tropical de Investigación y Enseñanza, Guia para el Manejo Integrado de Plagas en el cultivo del Tomate, Turrialba: CATIE, 1990.

[7] G. Karssen y M. Moens, «Root-knot nematodes,» de Plant Nematology, Cambridge, CABI, 2006, pp. 59-89.

[8] F. Ramírez, F. Chaverri, E. Cruz, C. Wesselling, L. Castillo y V. Bravo, Importación de Plaguicidas en Costa Rica: Periodo 2007-2009, Heredia: Universidad Nacional de Costa Rica, 2009.

[9] O. Fernández y A. Quesada, «https://www.sfe.go.cr,» Servicio Fitosanitario del Estado, 2013. [En línea]. Available: https://www.sfe.go.cr/tramites/Nematodos asociados a los Cultivos de CostaRica.pdf. [Último acceso: 20 febrero 2014].

[10] A. Esquivel, «Manual de identificación de géneros de nematodos importantes en Costa Rica,» Heredia, Universidad Nacional de Costa Rica, 2005, p. 50.

[11] K. Baker, «Nematode extraction and bioassays,» de Methodology, North Caroline University Graphics, 1985, pp. 19-23.

[12] W. Mai, P. Mullin, H. Lyon y K. Loeffler, Plant parasitic nematodes: a pictorial key to genera, Ithaca: Cornell University Press, 1996, p. 269.

[13] N. Marbán, Fitonematologia: Manual de Laboratorio, Turrialba: CATIE, 1987.

[14] B. Zuckerman, W. Mai y L. Krusberg, Plant Nematology Laboratory, México: Universidad Autónoma de Chapingo, 1990.

[15] Commonwealth Institute of Helminthology, Descriptions of Plant-parasitic Nematodes. CAB International, Wallingford, UK, Wallingford, UK: CAB International, 1972. 